\title{
Combined Diffusion Tensor and Magnetic Resonance Spectroscopic Imaging Methodology for Automated Regional Brain Analysis: Application in a Normal Pediatric Population
}

\author{
Nirmalya Ghosh ${ }^{a}$ Barbara Holshouser ${ }^{b}$ Udo Oyoyo $^{b}$ Stanley Barnes ${ }^{b}$ \\ Karen Tong $^{\text {b }}$ Stephen Ashwal ${ }^{a}$ \\ Departments of a Pediatrics and ${ }^{b}$ Radiology, Loma Linda University School of Medicine, Loma Linda, CA, USA
}

\section{Keywords}

Human brain maturation - Normal brain maturation . Magnetic resonance spectroscopy · Pediatric brain · Diffusion tensor imaging $\cdot$ Computational analysis

\begin{abstract}
During human brain development, anatomic regions mature at different rates. Quantitative anatomy-specific analysis of longitudinal diffusion tensor imaging (DTI) and magnetic resonance spectroscopic imaging (MRSI) data may improve our ability to quantify and categorize these maturational changes. Computational tools designed to quickly fuse and analyze imaging information from multiple, technically different datasets would facilitate research on changes during normal brain maturation and for comparison to disease states. In the current study, we developed a complete battery of computational tools to execute such data analyses that include data preprocessing, tract-based statistical analysis from DTI data, automated brain anatomy parsing from T1-weighted MR images, assignment of metabolite information from MRSI data, and co-alignment of these multimodality data streams for reporting of region-specific indices. We present statistical analyses of regional DTI and MRSI data in a cohort of normal pediatric subjects ( $n=72$; age range: $5-18$ years; mean $12.7 \pm 3.3$ years) to establish norma-
\end{abstract}

\section{KARGER}

(C) 2017 S. Karger AG, Basel

E-Mail karger@karger.com

www.karger.com/dne tive data and evaluate maturational trends. Several regions showed significant maturational changes for several DTI parameters and MRSI ratios, but the percent change over the age range tended to be small. In the subcortical region (combined basal ganglia [BG], thalami [TH], and corpus callosum $[C C])$, the largest combined percent change was a $10 \%$ increase in fractional anisotropy (FA) primarily due to increases in the BG (12.7\%) and $\mathrm{TH}(9 \%)$. The largest significant percent increase in $\mathrm{N}$-acetylaspartate (NAA)/creatine $(\mathrm{Cr})$ ratio was seen in the brain stem (BS) (18.8\%) followed by the subcortical regions in the BG (11.9\%), CC (8.9\%), and TH (6.0\%). We found consistent, significant $(p<0.01)$, but weakly positive correlations ( $r=0.228-0.329$ ) between NAA/Cr ratios and mean FA in the BS, BG, and CC regions. Age- and region-specific normative MR diffusion and spectroscopic metabolite ranges show brain maturation changes and are requisite for detecting abnormalities in an injured or diseased population.

(c) 2017 S. Karger AG, Basel

\section{Introduction}

Diffusion tensor imaging (DTI) and magnetic resonance spectroscopic imaging (MRSI) have proven invaluable in noninvasively examining maturational changes during normal development as well as in children with

Barbara Holshouser, $\mathrm{PhD}$

Loma Linda University Medical Center, MRI-B615

11234 Anderson Street

Loma Linda, CA 92354 (USA)

E-Mail bholshouser@llu.edu 
neurometabolic, genetic, and acquired disorders [1-14]. DTI of the pediatric population has been shown to provide quantitative measures of the spatial and temporal white matter (WM) development during the maturation process [3]. Numerous studies have shown that commonly measured DTI indices change rapidly from birth through infancy $[1,4,5]$ followed by slower changes through adolescence $[3,6-8]$. DTI studies have documented age-related regional variations, generally characterized overall by increasing fractional anisotropy (FA) and decreasing diffusivity with age. In contrast, MRSI measures specific brain metabolite concentrations that broadly reflect components of the neurovascular unit, such as neuronal and glial cell metabolism [15]. Similar to DTI, MRSI shows regional maturational changes with rapid changes in metabolite levels from birth through 24 months $[16,17]$ and much slower changes through adolescence [18-20]. When using DTI or MRSI it is necessary to take these maturational changes into account for studying pediatric brain diseases or injuries.

The complexity of neuroimaging data acquired with different MRI techniques warrants sophisticated postprocessing computational tools [21-23]. In DTI studies, three broad approaches have been adopted, either individually or in some combination: (1) voxel-based analysis (VBA) where individual voxels are compared in a registered common 3D coordinate space [24]; (2) atlas-based analysis that globally registers the entire brain with an atlas to obtain information on individual WM regions [8]; and (3) tract-based spatial statistics (TBSS) where DTI tracts (skeletons of WM regions) are registered with one tract atlas or template to quantify information for individual tracts [2, 25-28]. The requirement of registering each individual voxel in VBA has been shown to be too demanding in most cases [29] unless proper ground truth templates are generated [30]. Hence VBA is often utilized after atlas-based analysis or TBSS at the final comparison stage [31].

As part of an NIH-sponsored study on pediatric brain injuries, we developed a computational method to anatomically fuse and directly compare data from whole brain DTI and 3D MRSI acquisitions. We used TBSS followed by whole brain (not just WM) registration and anatomy-based analysis (not voxel or tract based), which may be more robust against eddy current and bias field effects in DTI data, because individual DTI data are not registered among themselves. In this paper, we report age- and region-specific normative diffusion and metabolite ranges in a pediatric population that are requisite for detecting abnormalities in an injured or diseased popula- tion. Children in our study ranged from 5 to 18 years of age, which, as described above, is a time when both DTI and MRSI brain maturational changes are subtle. Determining maturation profiles for commonly measured parameters in DTI and MRSI studies will allow us to determine whether age-specific evaluations must be made. The purpose of this paper is to describe the steps of our multimodality processing scheme (Fig. 1) and report age- and region-specific diffusion and metabolite ranges from healthy (normal) pediatric subjects using this method.

\section{Patients and Methods}

\section{Study Population}

Control subjects ( $n=72 ; 36$ males/36 females) between 5 and 18 years of age $($ mean $=12.7 \pm 3.3$ years; range: $5.4-18.5$ years $)$ were recruited as part of an Institutional Review Board-approved, NIHsponsored study on pediatric traumatic brain injuries (TBI). Written consent was obtained from the subject's parent or legal guardian and the subject if $>11$ years old. Assent was obtained from subjects between 7 and 11 years. Exclusion criteria included any MRI contraindications, neurological disorders, and previous brain trauma, or alcohol or drug abuse. All subjects underwent neuropsychological testing per study protocol to determine eligibility. Subjects were scanned without sedation, initially and 1 year later to match the protocol for TBI subjects. Data from both time points, if obtained, were included in the data reported in this study. However, 12 subjects did not return for their follow-up study at 1 year because they did not want to have another MRI $(n=3)$, or they moved out of the immediate area $(n=8)$. We took time to familiarize the children within the scanner environment to make them more comfortable before scanning. Images and spectra were checked for motion and repeated if necessary. However, in 3 subjects, the data for both MRSI and DTI were excluded due to excessive motion artifacts. These children did not return for follow-up studies. In an additional 2 studies, only the MRSI data were excluded due to motion artifacts.

\section{MRI Data Acquisition}

Whole-brain MRI and DTI were acquired on a 3-T whole-body imager (Trio/Tim; Siemens Medical Solutions, Erlangen, Germany) using a 12-channel receive-only head array coil. Imaging included an isotropic 3D sagittal T1-weighted inversion-prepared fast spoiled gradient echo sequence (MPRAGE, TR/TE $=1,950 / 2.26$ $\mathrm{ms}, \mathrm{NEX}=1$, voxel size $1.0 \times 1.0 \times 1.0 \mathrm{~mm}^{3}$ ) and a $3 \mathrm{D}$ sagittal T2weighted fast spin echo sequence with variable flip angles (SPACE, $\mathrm{TR} / \mathrm{TE}=3,200 / 415 \mathrm{~ms}, \mathrm{NEX}=1$, voxel size $1.0 \times 1.0 \times 1.0 \mathrm{~mm}^{3}$ ). DTI was acquired with a spin echo single-shot echo planar sequence using diffusion encoding in 30 noncollinear directions, $b$ values of 0 and $1,000 \mathrm{~s} / \mathrm{mm}^{2}, \mathrm{TR} / \mathrm{TE}=5,700 / 102 \mathrm{~ms}$, resolution $=$ $1.2 \times 1.2 \mathrm{~mm}^{2}$, and slice thickness $=3.0 \mathrm{~mm}$ with $0.9-\mathrm{mm}$ gap; 35 axial slices were oriented along a line drawn between the anterior and posterior commissures. Prior to DTI, 3D map shimming was performed. 
Fig. 1. Overall flow diagram of the multimodality information fusion methodology adopted in this work. The subject's highresolution $\mathrm{T} 1$ data were input into an atlasbased brain parcellation algorithm and segmented into tissue classes, white matter (WM), gray matter (GM), and cerebrospinal fluid (CSF), as well as 17 brain anatomic regions in T1/T2 space. The subject's diffusion and spectroscopy data were coaligned with their own T1/T2 data to transfer tissue and anatomy information to diffusion tensor imaging (DTI) and magnetic resonance spectroscopic imaging (MRSI) spaces. Finally, tract-based statistics from DTI and metabolite information from MRSI voxels were fused for individual brain anatomy and tissue regions to estimate normative developmental trends in a pediatric dataset. $\mathrm{AD}$, axial diffusivity; ADC, apparent diffusion coefficient; FA, fractional anisotropy; RD, radial diffusivity; TBSS, tract-based spatial statistics.

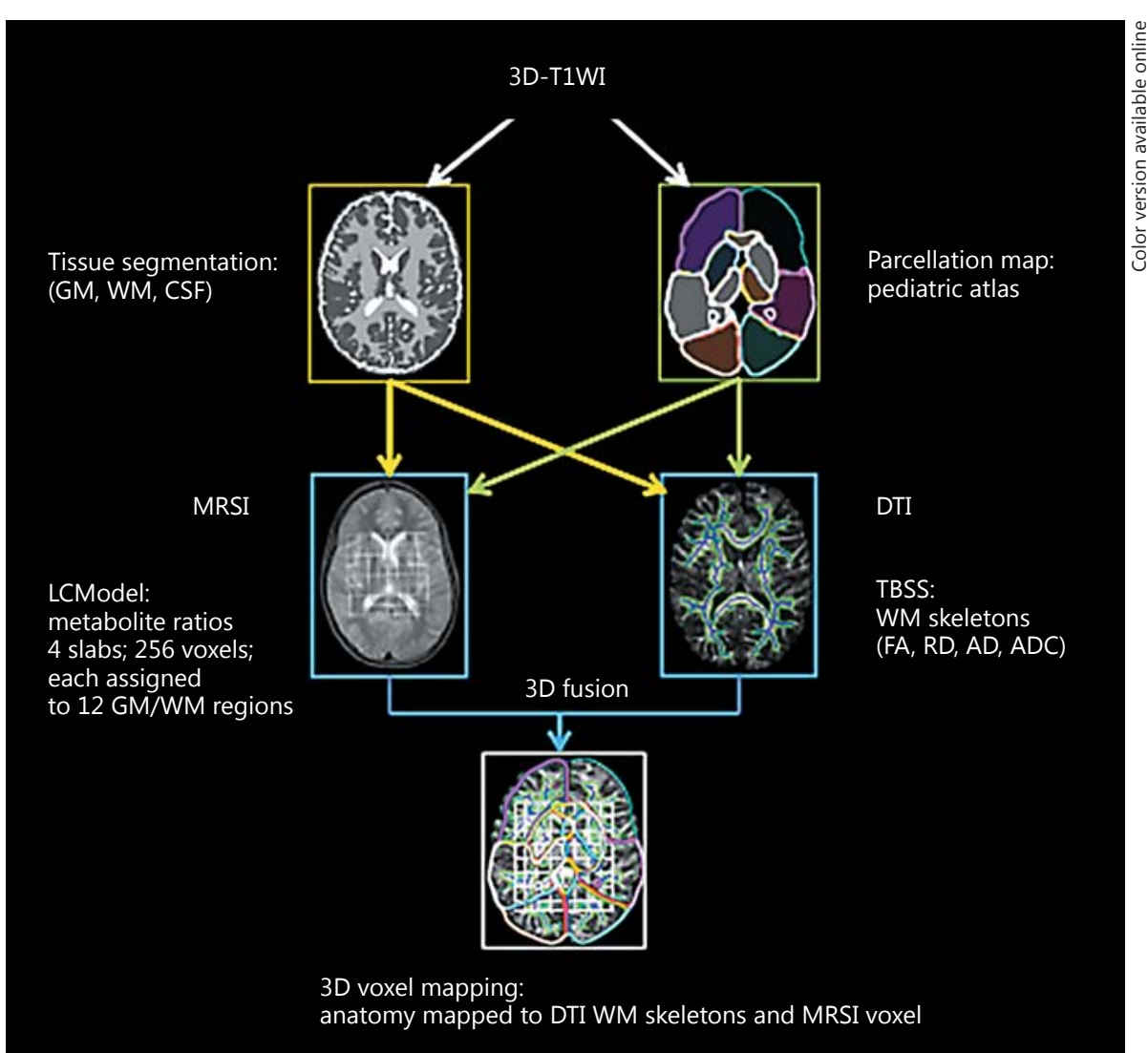

\section{MRSI Data Acquisition}

$3 \mathrm{D}$ proton MRSI was acquired using a water-suppressed pointresolved spectroscopy sequence with $\mathrm{TR} / \mathrm{TE}=1,700 / 144 \mathrm{~ms}$, $\mathrm{NEX}=1$, and 1,024 data points sampled with a dwell time of $1 \mathrm{~ms}$. Images from 3 orthogonal planes acquired during the imaging portion of the study were used for slab placement for MRSI and matched DTI orientation to facilitate direct comparison. Four or five multivoxel slabs, dependent on patient brain size, were acquired with the 3D MRSI (maximum of $160 \mathrm{~mm}$ field of view, $16 \times 16$ phase encodings, up to 64 voxels $[8 \times 8]$ volume of interest, and nominal voxel volume of $1 \mathrm{~cm}^{3}$ ) covering from the level of the mid-corpus callosum (CC) through the superior brain stem (BS), including portions of the frontal, parietal, temporal, and occipital WM, gray matter (GM), basal ganglia (BG), and thalami (TH).

\section{DTI Data Processing}

DTI Preprocessing. DTI data were preprocessed before tractbased statistical analysis was performed. The preprocessing steps were written in house and are detailed in the following subsections.

$3 D$ Interpolation. The manufacturer-provided DTI tensor files were interpolated to fill the interslice gap for TBSS and multimodality information fusion at a later stage. Due to time and clinical constraints, the DTI and MRI scans were not acquired at the same resolution. To produce a continuous volume of $3 \mathrm{D}$ data $(1.2 \times 1.2$ $\times 3 \mathrm{~mm}$ ), a $3 \mathrm{D}$ cubic interpolation routine developed in MATLAB version R2014a (Mathworks, Natick, MA, USA) was used for better matching of the DTI volume to the continuous T1WI volume for each of the tensor images $\left(\mathrm{D}_{x x}, \mathrm{D}_{x y}, \mathrm{D}_{x z}, \mathrm{D}_{y y}, \mathrm{D}_{y z}\right.$, and $\left.\mathrm{D}_{z z}\right)$ and the b0 datasets.

Skull Stripping Based on (bo) Diffusion Scan. The unweighted DTI ( $b=0 \mathrm{~mm}^{2} / \mathrm{s}$ or $\left.\mathrm{b} 0\right)$ images were used to extract brain area using a skull-stripping routine. All tensor and b0 images were coregistered by virtue of their acquisition and computation. Therefore, a binary mask of the skull-stripped brain from any of the 7 DTI volumes could be applied to others for stripping the skull. We found that the b0 images worked best for this purpose (Fig. 2a-d). Each slice in the 3D dataset was rescaled so that voxel values fell inside the $[0,1]$ range. A threshold of 0.005 was used to remove most of the background noise. For the remaining foreground (including the brain) regions, morphological cleaning (e.g., dilation, erosion, and filling internal holes in the foreground), and areabased filtering (i.e., removing foreground regions $<5 \%$ of the slice area) operations were performed using MATLAB routines to obtain a set of binary brain masks. The brain masks were then used to remove background noise and the skull in the DTI tensor images. All further DTI computations were performed only for the region defined by the binary brain mask. This process allowed for faster computation as the brain region consisted of only $\sim 35 \%$ of the entire 3D matrix.

DTI Map Computation. Skull-stripped tensor images were used to compute the following DTI parameter maps: FA, axial diffusivity $(\mathrm{AD})$, radial diffusivity $(\mathrm{RD})$, and apparent diffusion coefficient (ADC) maps based on standard formulas [32, 33]. 


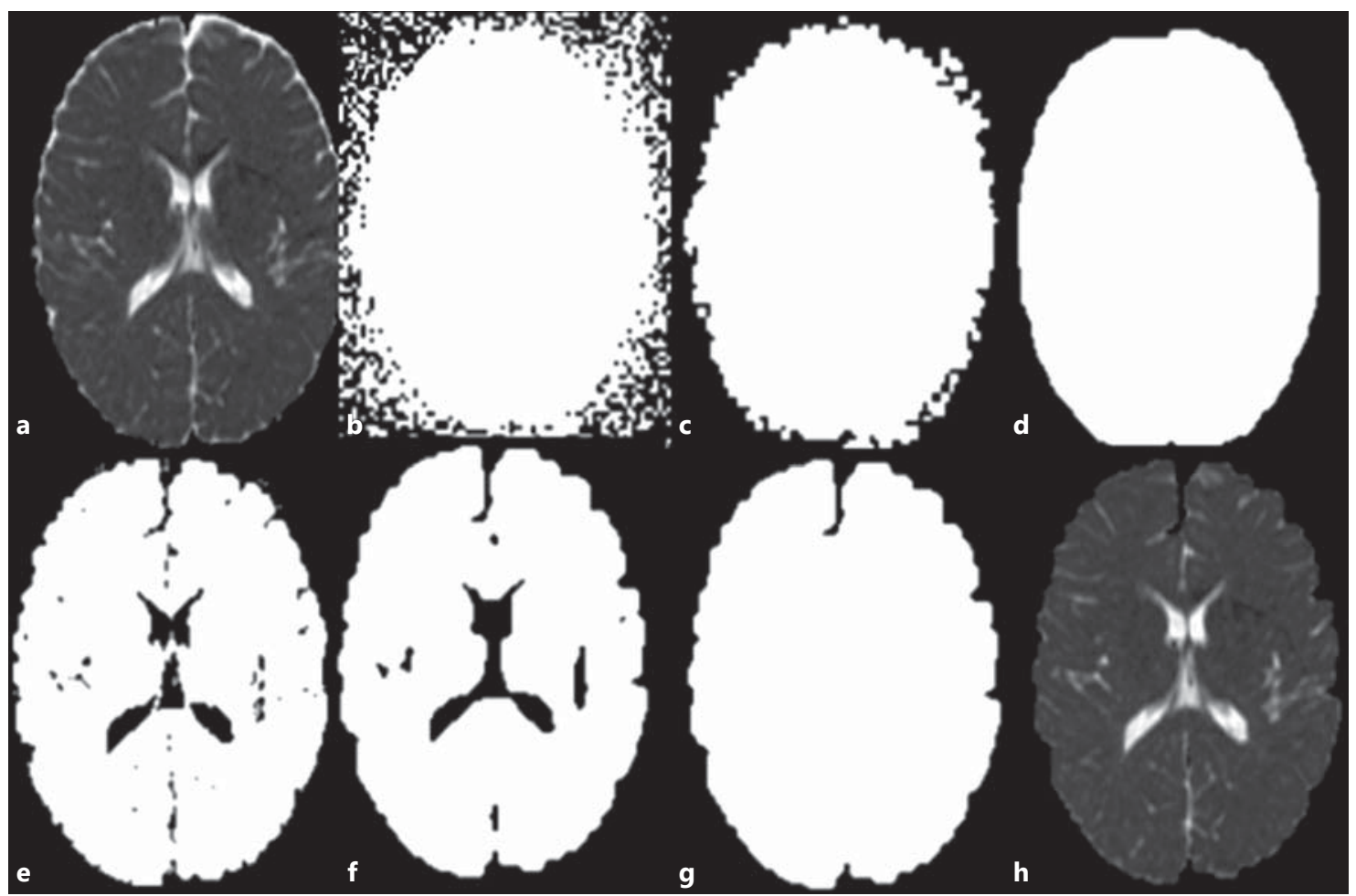

Fig. 2. Representative skull-stripping results from the b0 diffusion scan and CSF removal based on the apparent diffusion coefficient (ADC) map. Diffusion tensor imaging (DTI) b0 data (a) were first normalized within $[0,1]$ and binarized (b) using normalized intensity $>0.005$ followed by morphological opening, i.e., dilation followed by erosion, filling internal holes in the foreground (white), and area-based filtering, i.e., removing regions $<5 \%$ of DTI acquisition matrix size performed in sequence to obtain a final skullstripped DTI brain mask (c). This mask was used for other tensor

Cerebrospinal Fluid Removal Based on ADC Maps. Cerebrospinal fluid (CSF) masking was performed using ADC maps to identify high signal intensity voxels to produce a final binary brain mask which was applied to other DTI maps to improve tract-based analysis. ADC maps were used to detect CSF voxels using an ADC threshold $>500 \times 10^{-5} \mathrm{~mm}^{2} / \mathrm{s}$. After morphological cleaning (erosion, dilation, majority filtering, and area-based filtering), voxels from CSF outside the brain were eliminated from the binary mask while the ventricular voxels were still included (Fig. $2 \mathrm{e}-\mathrm{h}$ ). This provided the final brain mask used to clean the remaining DTI maps. If needed, additional skull stripping was performed during this step using an ADC threshold of $<5 \times 10^{-5} \mathrm{~mm}^{2} / \mathrm{s}$, followed by additional morphological cleaning.

\section{TBSS from DTI}

In our TBSS method, the physical locations of the skeleton are the central voxels within a WM tract, and the values assigned to each skeletal voxel change depending on which statistical parameter is being considered. TBSS processing steps were written in house and detailed in the following subsections. images and for the rest of the methods. DTI-b0-based skullstripped ADC obtained from $\mathrm{c}$ was binarized by an ADC threshold $\left(>5 \times 10^{-5} \mathrm{~mm}^{2} / \mathrm{s}\right)$, morphologically cleaned, and small outlier regions were removed by area-based filtering $(\mathbf{d})$. CSF was removed using an ADC threshold $\left(<500 \times 10^{-5} \mathrm{~mm}^{2} / \mathrm{s}\right)(\mathbf{e})$, further morphologically cleaned (f), and the ventricle was reinstated into the DTI brain mask (g) to get the final skull-/CSF-stripped ADC map (h). The final brain mask (g) was used to clean the rest of the DTI maps before further processing.

Finding WM Masks and Skeletons from FA Maps. WM brain tracts were extracted (including peripheral WM tracts), and a skeleton was computed by using an FA threshold and iterative thinning of WM. Binary masks were obtained using a specific threshold of FA $>0.2$ on our 2D FA maps (Fig. 3a) because testing determined that this threshold best defined the WM of the brain. This process produced some outlier regions (Fig. 3b) that were removed by morphological cleaning (Fig. 3c). Finally, a region-based connected component analysis found small nonconnected outlier regions $<20$ voxels in size which were then removed using area-based filtering. Using a MATLAB routine for medial axis computation (MATLAB function "bwmorph [BinaryMask, 'skel', inf]") until the 2D skeletal connectivity was preserved, a skeleton of the WM mask was computed for every FA map by iteratively removing boundary pixels of the WM binary mask (Fig. 3c) to produce a single connected skeleton for each WM region (Fig. 3d). The rationale for using FA $>0.2$ for WM is shown in Fig. 3e-h illustrating that smaller FA thresholds overestimate WM regions while larger FA thresholds underestimate them.

Finding Perpendicular Lines to the Skeletal Voxels. To find the perpendicular line for a particular skeletal voxel $(V)$ with $2 \mathrm{D}$ location 


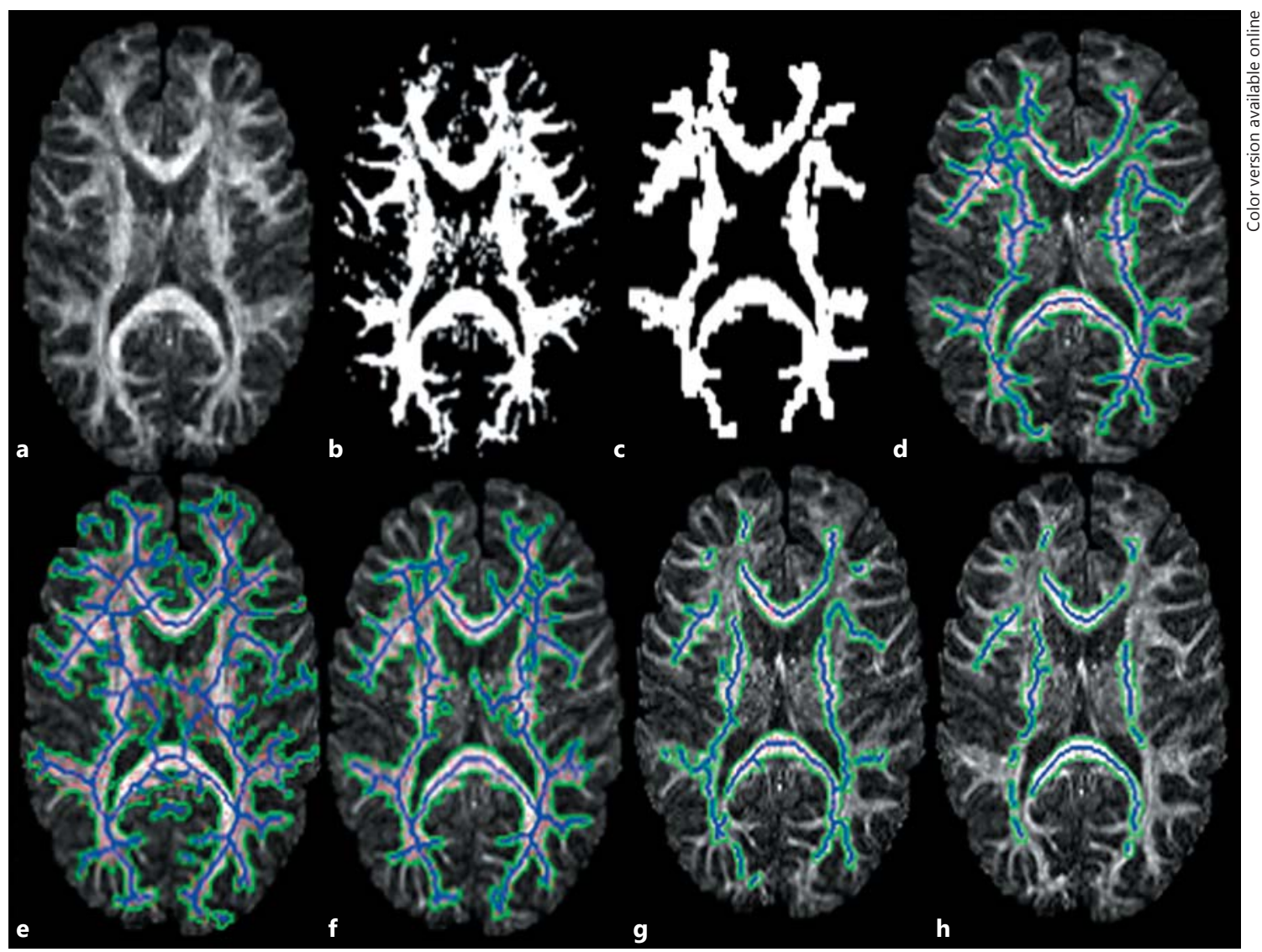

Fig. 3. Illustration of fractional anisotropy (FA)-based detection of white matter (WM) region and corresponding skeleton. Skull- and CSF-stripped FA map (a) was binarized (b) with thresholding (FA $>0.2$ ) and morphologically cleaned to obtain WM mask region c, from which FA skeleton (blue), WM perimeter (green), and regional maxima (red) were computed (d). The WM regions and skeleton from the FA map were used for the remaining diffusion tensor imaging maps for statistical analysis. Rationale for using FA $>0.2$ for detecting WM is illustrated in the bottom row. Smaller FA thresholds of FA $>0.1$ (e) or FA $>0.15$ (f) overestimated the WM regions, while larger thresholds of FA $>0.25$ (g) or FA $>0.3$ (h) underestimated them compared to FA $>0.2$ (d), which performed better and hence was utilized in the current study. $\left(x_{v}, y_{v}\right)$, the neighborhood structure (10 skeletal voxels on each side of $V$ along the skeleton) was fitted to a 5 th-degree polynomial curve. This fitted curve of the skeleton was then used to first calculate lines tangential to it and then to calculate the lines perpendicular to it at the location $V$. The voxels along the perpendicular line that were also located within the WM binary mask (Fig. 3c) were considered for computing the statistical measure for the skeletal voxel $V$ in the next step. This method was followed for all skeleton voxels.

Defining Skeleton Values. The skeletons represent the center of the WM tracts. However, we replaced the values in the skeleton voxels with several specific statistical measures (mean, median, maximum, and mode) of the voxel values on the line perpendicular to the skeleton for each DTI index. The WM binary mask and skeleton derived from the FA maps were superimposed onto other DTI maps (RD, AD, and ADC). The perpendicular line was computed for every skeletal voxel in each DTI map, and the values along that line were collected. The mean, median, maximum, and mode of these values were calculated and assigned to the corre- sponding skeletal voxel. The mean values (except FA, where maxFA is also reported) are reported in this paper. To obtain a single volumetric statistic for each subject, we computed the mean and standard deviation of each statistical measure and DTI index for the entire interpolated 3D brain (total).

\section{Brain Anatomy Detection}

DTI is not the optimal modality for brain anatomy detection. For this, T1WI data were used, and the following steps were followed and detailed in the following subsections.

Segmentation. Segmentation routines were used to compute the percentage of each voxel in GM, WM, and CSF using T1WI data with the Statistical Parameter Mapping (SPM5) software (The Wellcome Department of Imaging Neuroscience, Institute of Neurology, University College London, London, UK) (http:// www.fil.ion.ucl.ac.uk/spm/). Separate binary masks of these regions were saved for each brain T1WI slice. These data were used later for information fusion. 
Fig. 4. Examples of automated brain parsing and overlay of magnetic resonance spectroscopic imaging (MRSI) data on diffusion tensor imaging (DTI) space. a-c Regional boundaries of 17 different regions are color coded and superimposed on corresponding DTI b0 data for visualization. These regions were utilized for region-specific statistics of different DTI parameters. d, e A mean value for each DTI parameter was calculated for each MRSI voxel based on the DTI skeleton pixel values within the MRSI voxel. The skeleton pixels located within each MRSI voxel can represent different statistics (mean, median, maximum, and mode) for different DTI metrics (axial diffusivity, fractional anisotropy, radial diffusivity, and mean diffusivity) calculated from pixels located along perpendicular lines to the skeleton.

Table 1. Individual and aggregate brain regions used for comparisons
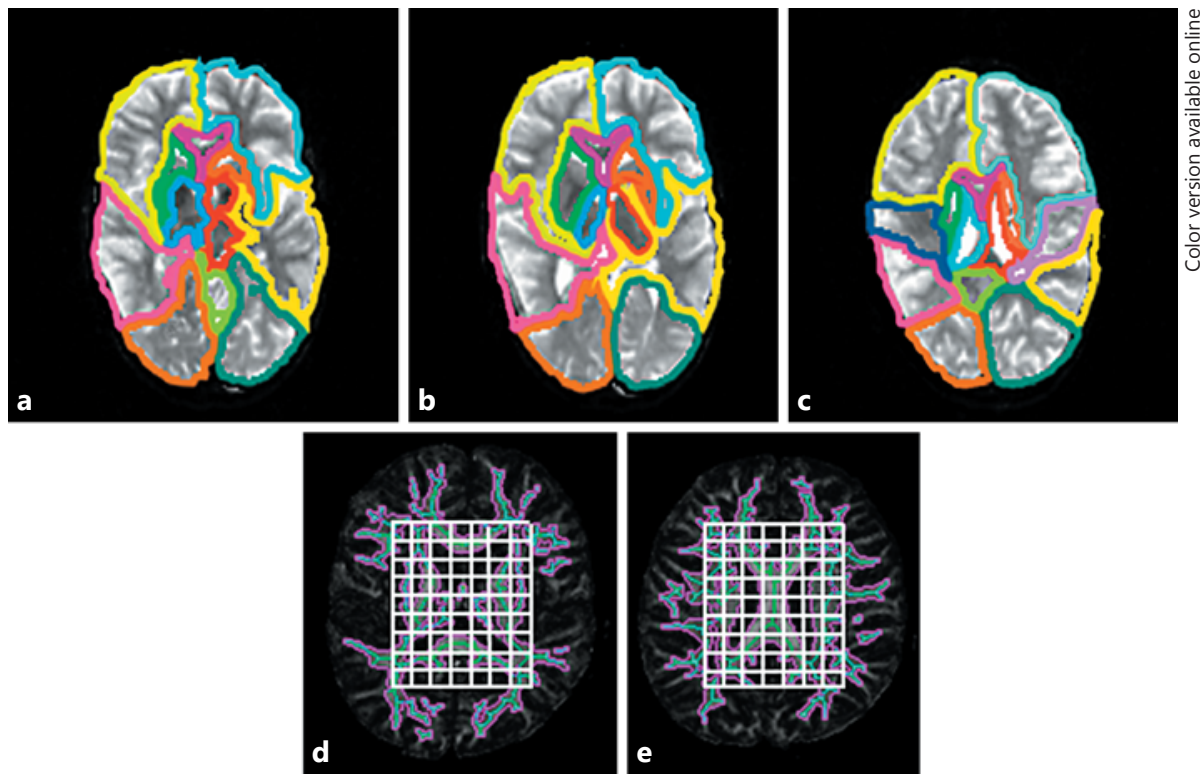

\begin{tabular}{lll}
\hline Superficial brain (SB) & Subcortical brain (SC) & Posterior fossa (PF) \\
\hline $\begin{array}{ll}\text { Frontal white matter (FWM) } \\
\text { Frontal gray matter (FGM) }\end{array}$ & $\begin{array}{l}\text { Basal ganglia (BG) } \\
\text { Corpus callosum (CC) }\end{array}$ & $\begin{array}{l}\text { Brain stem/pons (BS) } \\
\text { Cerebellum (CB) }\end{array}$ \\
$\begin{array}{l}\text { Parietal white matter (PWM) } \\
\text { Parietal gray matter (PGM) }\end{array}$ & & \\
Temporal white matter (TWM) & & \\
Temporal gray matter (TGM) & & \\
\hline
\end{tabular}

Brain Parsing/Labeling. We determined brain anatomic regions from the T1WI data and co-registered DTI b0 scan volumes of each subject to their own T1WI volume and their corresponding anatomic regions obtained in the step "TBSS from DTI." To do this, an established pediatric brain parsing pipeline with the Laboratory of Neuroimaging (LONI) brain Parser software from the University of Southern California, Los Angeles, CA, USA (http:// www.pipeline.loni.usc.edu) (namely "BrainParser [Pediatric Atlas]") was modified to handle anisotropic pixel size of our T1WI data, as well as to optimize internal operational parameters, e.g., virtual memory and stack size parameters in "Syn" and "SSMA" modules for our specific purpose and used to label 83 T1-derived anatomic brain regions. Our modified LONI pediatric pipeline is shown in online supplementary Figure 1 (for all online suppl. material, see www.karger.com/doi/10.1159/000475545).

Co-Registering DTI $b 0$ and T1WI. Co-registration was performed between the b0 dataset from DTI (effectively T2-weighted images) and T1WI so that for each voxel, T1-based tissue classes and anatomic regions (from the steps described in the two preceding paragraphs) could be subsequently utilized for fusion of DTI and MRSI data to directly compare measured parameters from both. Co-registration of the b0 volume and T1WI volume and subsequently translating T1 space anatomy to DTI space was performed by another LONI workflow (online suppl. Fig. 2) developed from the available pipeline modules. Using MATLAB, the 83 regions were then merged into 17 regions to directly compare developmental trends and comparisons between lower-resolution MRSI data and DTI data (Fig. $4 \mathrm{a}-\mathrm{c}$ ). Left and right regions were then merged into one region to finally produce 12 anatomic regions and 3 aggregate regions used for comparisons between DTI and MRSI parameters (Table 1).

\section{MRSI Data Processing}

MRSI spectra were postprocessed using LCModel (version 6.0; Stephen Provencher Inc., Oakville, ON, Canada), which allows quantification of the metabolites from the in vivo data. LCModel is a well-established, commercially available, automatic (user-independent), time domain fitting routine that employs a basic set of concentration-calibrated model spectra of individual metabolites to estimate the absolute concentrations of similar brain metabolites from the in vivo spectral data correcting for residual eddy current effects. For each MRSI voxel, individual metabolite concentrations are reported with a confidence measurement (SD\%) reflecting maximum-likelihood estimates and their uncertainties 
Table 2. Metabolite ratio and diffusion tensor imaging (DTI) parameter regression analyses with age

\begin{tabular}{|c|c|c|c|c|c|c|c|c|}
\hline Region & & $\mathrm{NAA} / \mathrm{Cr}$ & NAA/Cho & $\mathrm{Cho} / \mathrm{Cr}$ & Mean FA & $\mathrm{ADC}$ & $\mathrm{AD}$ & $\mathrm{RD}$ \\
\hline \multirow[t]{3}{*}{ BS } & & $\mathrm{F}_{2,79}=8.7$ & $\mathrm{~F}_{2,79}=12.9$ & $\mathrm{~F}_{2,80}=3.1$ & $\mathrm{~F}_{2,73}=0.4$ & $\mathrm{~F}_{2,75}=4.3$ & $\mathrm{~F}_{2,76}=3.7$ & $\mathrm{~F}_{2,76}=4.5$ \\
\hline & $p$ & $<0.001$ & $<0.001$ & 0.04 & ns & 0.02 & 0.03 & 0.014 \\
\hline & $\Delta$ & $18.8 \%$ & $23.4 \%$ & $-7.3 \%$ & ns & $-7.7 \%$ & $-8.2 \%$ & $-8.9 \%$ \\
\hline \multirow[t]{3}{*}{$\overline{\mathrm{BG}}$} & & $\mathrm{F}_{2,89}=9.4$ & $\mathrm{~F}_{2,87}=17.2$ & $\mathrm{~F}_{2,88}=4.5$ & $\mathrm{~F}_{2,89}=11.3$ & $\mathrm{~F}_{2,89}=3.6$ & $\mathrm{~F}_{2,89}=2.1$ & $\mathrm{~F}_{2,88}=8.3$ \\
\hline & $p$ & $<0.001$ & $<0.001$ & 0.014 & $<0.001$ & 0.03 & $\mathrm{~ns}$ & 0.001 \\
\hline & $\Delta$ & $11.9 \%$ & $18.1 \%$ & $-9.3 \%$ & $12.7 \%$ & $-3.8 \%$ & ns & $-9.5 \%$ \\
\hline \multirow[t]{3}{*}{$\overline{\mathrm{CC}}$} & & $\mathrm{F}_{2,87}=5.9$ & $\mathrm{~F}_{2,88}=8.8$ & $\mathrm{~F}_{2,87}=4.5$ & $\mathrm{~F}_{2,87}=1.03$ & $\mathrm{~F}_{2,88}=13.1$ & $\mathrm{~F}_{2,87}=7.3$ & $\mathrm{~F}_{2,87}=12.4$ \\
\hline & $p$ & 0.004 & $<0.001$ & 0.014 & ns & $<0.001$ & 0.001 & $<0.001$ \\
\hline & $\Delta$ & $8.9 \%$ & $15.5 \%$ & $-9.3 \%$ & ns & $-10.3 \%$ & $-7.3 \%$ & $-15.1 \%$ \\
\hline \multirow[t]{3}{*}{$\overline{\mathrm{TH}}$} & & $\mathrm{F}_{2,89}=4.2$ & $\mathrm{~F}_{2,89}=8.7$ & $\mathrm{~F}_{2,88}=5.5$ & $\mathrm{~F}_{2,89}=3.2$ & $\mathrm{~F}_{2,89}=0.2$ & $\mathrm{~F}_{2,88}=0.7$ & $\mathrm{~F}_{2,89}=0.3$ \\
\hline & $p$ & 0.018 & $<0.001$ & 0.006 & 0.047 & ns & ns & ns \\
\hline & $\Delta$ & $6.0 \%$ & $13.5 \%$ & $-8.9 \%$ & $9.2 \%$ & ns & ns & ns \\
\hline \multirow[t]{3}{*}{ FGM } & & $\mathrm{F}_{2,88}=0$ & $\mathrm{~F}_{2,88}=2.2$ & $\mathrm{~F}_{2,86}=4.5$ & & & & \\
\hline & $p$ & $\mathrm{~ns}$ & ns & 0.013 & & & & \\
\hline & $\Delta$ & ns & ns & $-5.9 \%$ & & & & \\
\hline \multirow[t]{3}{*}{ FWM } & & $\mathrm{F}_{2,89}=0.9$ & $\mathrm{~F}_{2,89}=7.4$ & $\mathrm{~F}_{2,87}=6.8$ & $\mathrm{~F}_{2,89}=5.1$ & $\mathrm{~F}_{2,86}=21.1$ & $\mathrm{~F}_{2,87}=23.1$ & $\mathrm{~F}_{2,88}=11.7$ \\
\hline & $p$ & ns & 0.001 & 0.002 & 0.008 & $<0.001$ & $<0.001$ & $<0.001$ \\
\hline & $\Delta$ & ns & $19.2 \%$ & $-10.8 \%$ & $4.4 \%$ & $-10.1 \%$ & $-8.8 \%$ & $-11.7 \%$ \\
\hline \multirow[t]{3}{*}{$\overline{\mathrm{OGM}}$} & & $\mathrm{F}_{2,74}=1.2$ & $\mathrm{~F}_{2,75}=3.5$ & $\mathrm{~F}_{2,76}=3.8$ & & & & \\
\hline & $p$ & ns & 0.035 & 0.026 & & & & \\
\hline & $\Delta$ & ns & $13.6 \%$ & $-14.6 \%$ & & & & \\
\hline \multirow[t]{3}{*}{ PWM } & & $\mathrm{F}_{2,86}=2.7$ & $\mathrm{~F}_{2,85}=9.7$ & $\mathrm{~F}_{2,85}=6.7$ & $\mathrm{~F}_{2,84}=1.3$ & $\mathrm{~F}_{2,85}=5.4$ & $\mathrm{~F}_{2,83}=8.5$ & $\mathrm{~F}_{2,86}=2.1$ \\
\hline & $p$ & ns & $<0.001$ & 0.002 & ns & 0.006 & $<0.001$ & $\mathrm{~ns}$ \\
\hline & $\Delta$ & ns & $19.2 \%$ & $-10.8 \%$ & ns & $-5.9 \%$ & $-7.2 \%$ & ns \\
\hline \multirow[t]{3}{*}{$\overline{P G M}$} & & $\mathrm{~F}_{2,85}=0.1$ & $\mathrm{~F}_{2,85}=2.7$ & $\mathrm{~F}_{2,86}=3.2$ & & & & \\
\hline & $p$ & ns & ns & 0.046 & & & & \\
\hline & $\Delta$ & ns & ns & $-7.9 \%$ & & & & \\
\hline \multirow[t]{3}{*}{ TWM } & & $\mathrm{F}_{2,89}=0.3$ & $\mathrm{~F}_{2,88}=5.6$ & $\mathrm{~F}_{2,89}=7.1$ & $\mathrm{~F}_{2,87}=11.4$ & $\mathrm{~F}_{2,89}=31.1$ & $\mathrm{~F}_{2,89}=21.2$ & $\mathrm{~F}_{2,88}=27.6$ \\
\hline & $p$ & ns & 0.005 & 0.001 & $<0.001$ & $<0.001$ & $<0.001$ & $<0.001$ \\
\hline & $\Delta$ & ns & $11.6 \%$ & $-9.9 \%$ & $4.8 \%$ & $8.8 \%$ & $-6.1 \%$ & $-12.6 \%$ \\
\hline \multirow[t]{2}{*}{$\overline{\mathrm{PF}}$} & $p$ & $\begin{array}{l}\mathrm{F}_{2,80}=8.7 \\
<0.001\end{array}$ & $\begin{array}{l}\mathrm{F}_{2,80}=5.5 \\
0.006\end{array}$ & $\begin{array}{l}\mathrm{F}_{2,79}=1.4 \\
\mathrm{~ns}\end{array}$ & $\begin{array}{l}\mathrm{F}_{2,89}=11.3 \\
<0.00\end{array}$ & $\begin{array}{l}\mathrm{F}_{2,75}=4.3 \\
0.02\end{array}$ & $\begin{array}{l}\mathrm{F}_{2,76}=3.7 \\
0.03\end{array}$ & $\begin{array}{l}\mathrm{F}_{2,76}=4.5 \\
0.014\end{array}$ \\
\hline & $\Delta$ & $19.1 \%$ & $15.3 \%$ & ns & $12.7 \%$ & $-7.7 \%$ & $-8.2 \%$ & $-8.9 \%$ \\
\hline \multirow[t]{2}{*}{$\overline{\mathrm{SC}}$} & $p$ & $\begin{array}{l}\mathrm{F}_{2,89}=14.2 \\
<0.001\end{array}$ & $\begin{array}{l}\mathrm{F}_{2,88}=20.1 \\
<0.001\end{array}$ & $\begin{array}{l}\mathrm{F}_{2,87}=8.8 \\
<0.001\end{array}$ & $\begin{array}{l}\mathrm{F}_{2,89}=21.5 \\
<0.001\end{array}$ & $\begin{array}{l}\mathrm{F}_{2,88}=1.5 \\
\mathrm{~ns}\end{array}$ & $\begin{array}{l}\mathrm{F}_{2,87}=1.3 \\
\text { ns }\end{array}$ & $\begin{array}{l}\mathrm{F}_{2,88}=4.4 \\
0.015\end{array}$ \\
\hline & $\Delta$ & $10.9 \%$ & $17.7 \%$ & $-10.4 \%$ & $10.9 \%$ & ns & ns & $-5.7 \%$ \\
\hline \multirow[t]{3}{*}{$\overline{\mathrm{SB}}$} & & $\mathrm{F}_{2,89}=4.7$ & $\mathrm{~F}_{2,88}=14.8$ & $\mathrm{~F}_{2,89}=7.2$ & $\mathrm{~F}_{2,85}=4.1$ & $\mathrm{~F}_{2,88}=22.8$ & $\mathrm{~F}_{2,88}=18.9$ & $F_{2,87}=16.1$ \\
\hline & $p$ & 0.011 & $<0.001$ & 0.001 & ns & $<0.001$ & $<0.001$ & $<0.001$ \\
\hline & $\Delta$ & $9.7 \%$ & $15.1 \%$ & $-7.3 \%$ & ns & $-9.7 \%$ & $-8.3 \%$ & $-10.8 \%$ \\
\hline
\end{tabular}

F statistic and $p$ value reported from multiple regression analyses. $\Delta, \%$ change from 5 to 18 years. CB and TGM regions (not listed) showed no significant differences (ns, nonsignificant: $p \geq 0.05$ ). AD, axial diffusivity; ADC, apparent diffusion coefficient; Cho, choline; $\mathrm{Cr}$, creatine; FA, fractional anisotropy; NAA, N-acetylaspartate; RD, radial diffusivity. See Table 1 for further abbreviations.

(Cramer-Rao lower bounds). Metabolite concentrations with an $\mathrm{SD} \%$ of $\leq 10$ were included in this study. Concentrations for $\mathrm{N}$ acetyl-aspartate (NAA; $2.02 \mathrm{ppm})$, total creatine (Cr; $3.02 \mathrm{ppm})$, total choline (Cho; $3.20 \mathrm{ppm}$ ), and lactate, if present (identified as an inverted doublet at $1.33 \mathrm{ppm}$ and $7-\mathrm{Hz}$ splitting), were measured for each voxel that met the above criteria. Peak concentrations for each voxel were recorded in a spreadsheet for further analysis and used to calculate metabolite ratios (NAA/Cr, NAA/ Cho, Cho/Cr, and lactate/Cr).

DTI and MRSI Brain Analysis in Normal Children
Finding DTI Parameters, Tissue Types, and Anatomy for Each MRSI Voxel

The 3D MRSI grid was manually oriented during scanning to cover relevant brain regions from above the CC through the top of the BS. The 3D location and orientation parameters in the MRSI data header were used to find anatomic correspondence between MRI and MRSI voxels. In-house software co-registered the 3D MRSI slabs with 3D T1WI/T2WI for assigning the anatomic location of the voxel and the segmented 3D T1WI to estimate the per-

Dev Neurosci 2017;39:413-429 


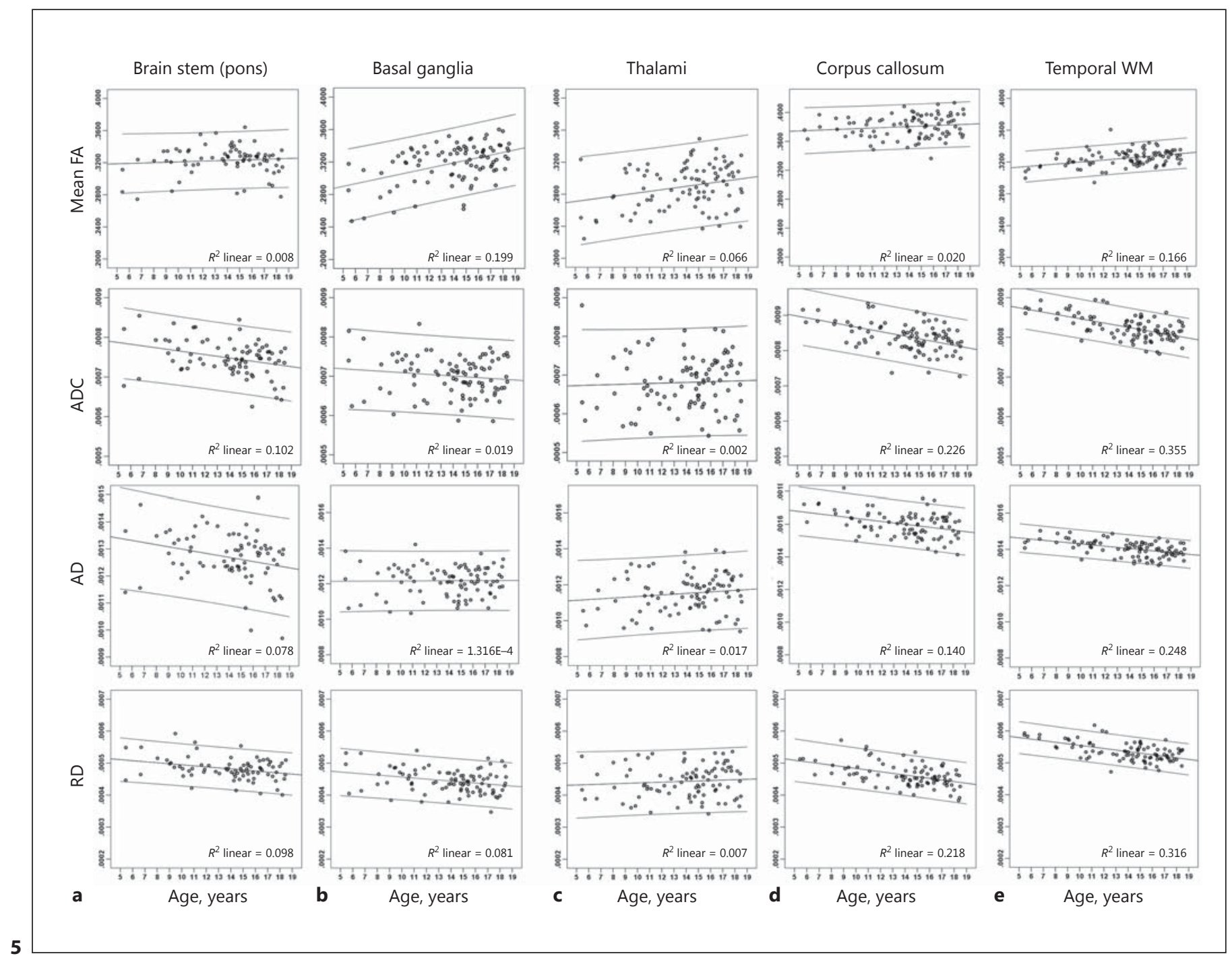

(Figure continued on next page.)

cent CSF, WM, and GM for each voxel. To limit partial volume errors, only voxels with $>80 \% \mathrm{WM}$ or GM were included in the analysis, and voxels with $>50 \%$ CSF were excluded. This software was developed in our laboratory incorporating routines from MATLAB and SPM5. MRSI voxels from the frontal, parietal, and temporal regions were assigned as GM or WM resulting in data from a total of 12 anatomic regions listed in Table 1.

The MRSI grid for each slab was also overlaid onto 3D DTI skeleton masks to obtain DTI parameters (mean FA, AD, RD, and ADC) of each voxel for comparison to metabolite ratios (Fig. 4d, e). The assigned anatomic regions and DTI results for each voxel were saved, and mean values for each MRSI ratio and DTI parameter (WM and deep GM only) were then calculated for the spectroscopy-defined individual regions. Mean values for measured parameters were also calculated for the following aggregate regions: superficial brain; subcortical brain; posterior fossa; and total (all regions), as defined in Table 1.

\section{Statistical Analysis}

We performed multiple regression analyses (SPSS 22, Chicago, IL, USA) with age and gender as covariates to determine whether age-related changes in DTI parameters and MRSI metabolite ratios were present. These changes were considered to be statistically significant when the regression analyses yielded slopes that were significantly different from zero at a level of $p<0.05$. To quantify possible outlier values, studentized deleted residuals from mean FA, ADC, AD, RD, and NAA/Cr, NAA/Cho, and Cho/Cr ratios were calculated. Regression analyses were performed again after outlier analysis, excluding individuals exceeding studentized deleted residuals \pm 3 to make sure that these subjects did not overly affect the results. A histogram with superimposed normal curve and a P-P probability plot were also used to assess normality for changes in DTI parameters and MRSI metabolite ratios with age.

All variables were assessed for normality using the Kolmogorov-Smirnov and Shapiro-Wilks procedures. We found that all variables were normally distributed. We did not find significant 


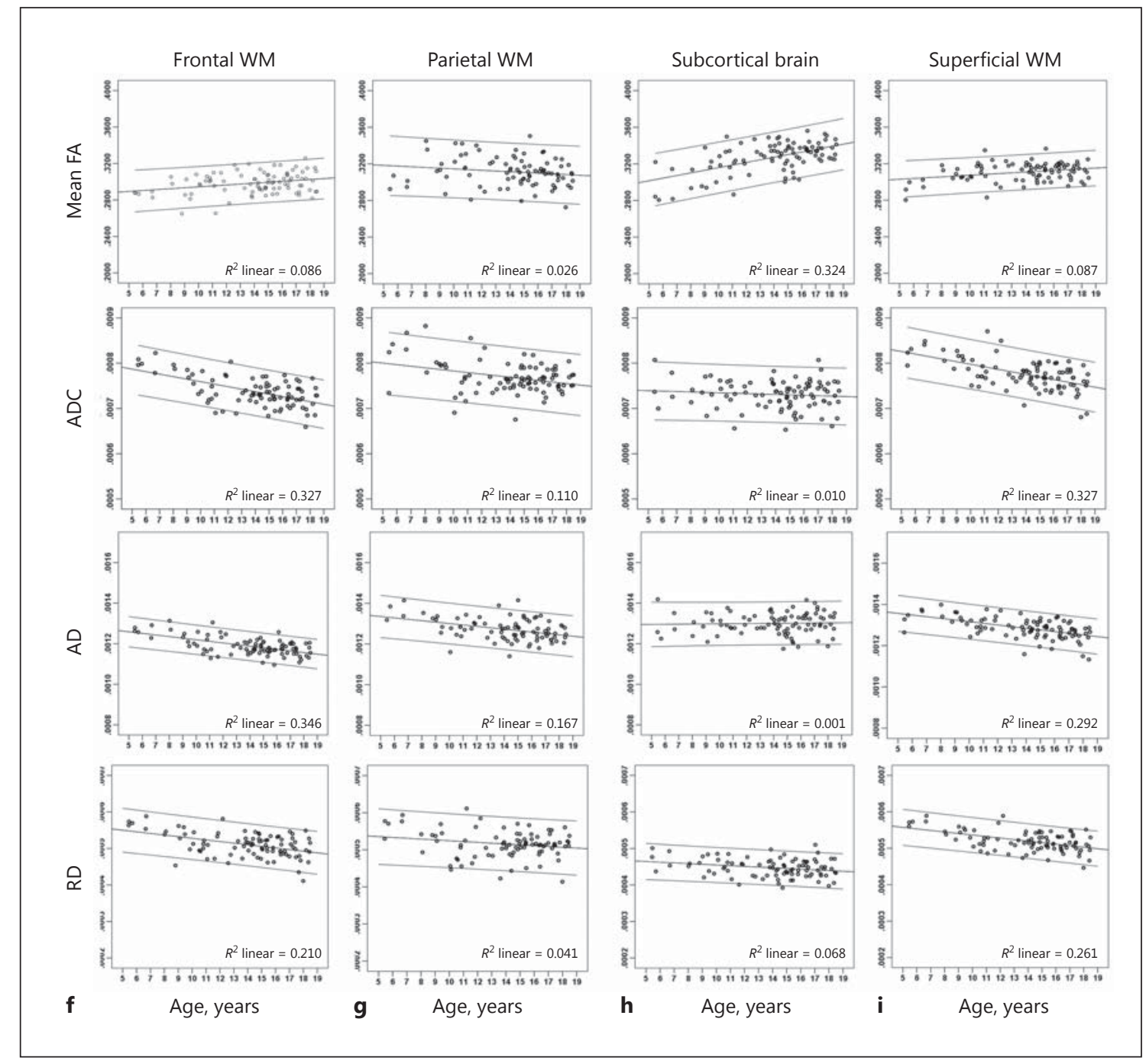

Fig. 5. Measured diffusion tensor imaging parameters for mean fractional anisotropy (FA), mean apparent diffusion coefficient (ADC), mean axial diffusivity (AD), and mean radial diffusivity (RD) for 7 brain regions and 2 combined regions; subcortical (basal ganglia + thalami + corpus callosum) and superficial brain (temporal + parietal + frontal white matter $[\mathrm{WM}])$ versus age in years. The linear regression fits, $R^{2}$ value, and $95 \%$ confidence intervals are shown.

left versus right differences between MRSI or DTI parameters that were considered clinically significant (i.e., $>2$ SD). To determine whether DTI parameters were linearly related to metabolite ratios, Pearson correlation coefficients were calculated.

An outcome-based method was used to determine an optimal cutoff for age regarding DTI and MRSI parameters using SAS 9.3 (SAS Institute Inc., Cary, NC, USA). Given the absence of a priori clinical information regarding the covariate relationship between age and MRSI values, a cutoff threshold of 12 years of age was determined empirically with graphical methods including grouped data plots and Martingale residuals used to investigate the lack of model fit. In addition, a twofold cross-validation technique was used to determine the statistical significance of the cutoff threshold

DTI and MRSI Brain Analysis in Normal Children of 12 years of age that included the steps of randomly assigning all observations into either of two datasets of approximately equal size $(n=24)$. Using similar methods, no reliable age stratification threshold was observed for DTI parameters.

\section{Results}

\section{Normal Developmental Patterns of DTI Parameters}

DTI parameter changes with age are shown in graphs for individual and combined regions with linear fits, con-

Dev Neurosci 2017;39:413-429 


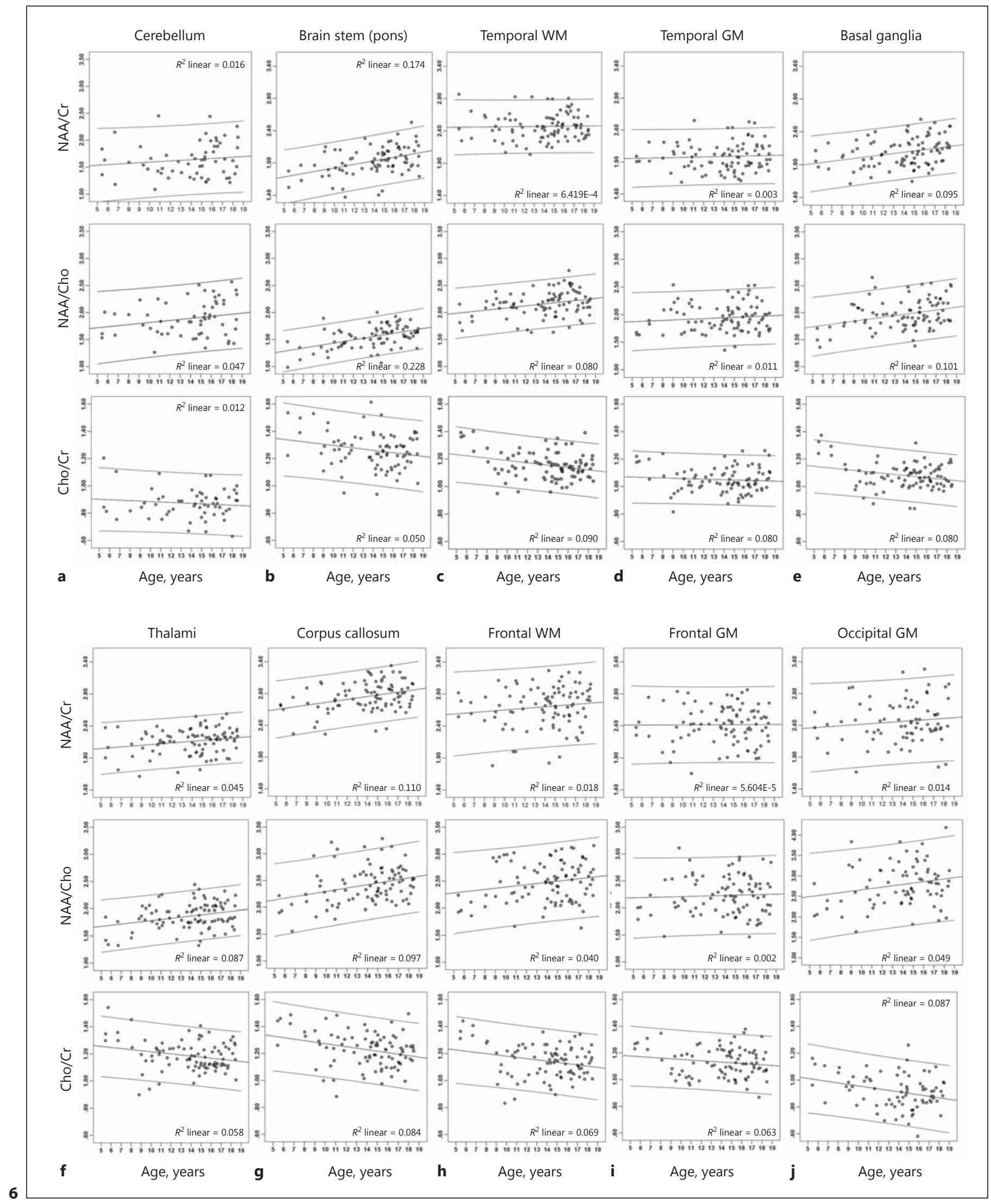

(Figure continued on next page.) 


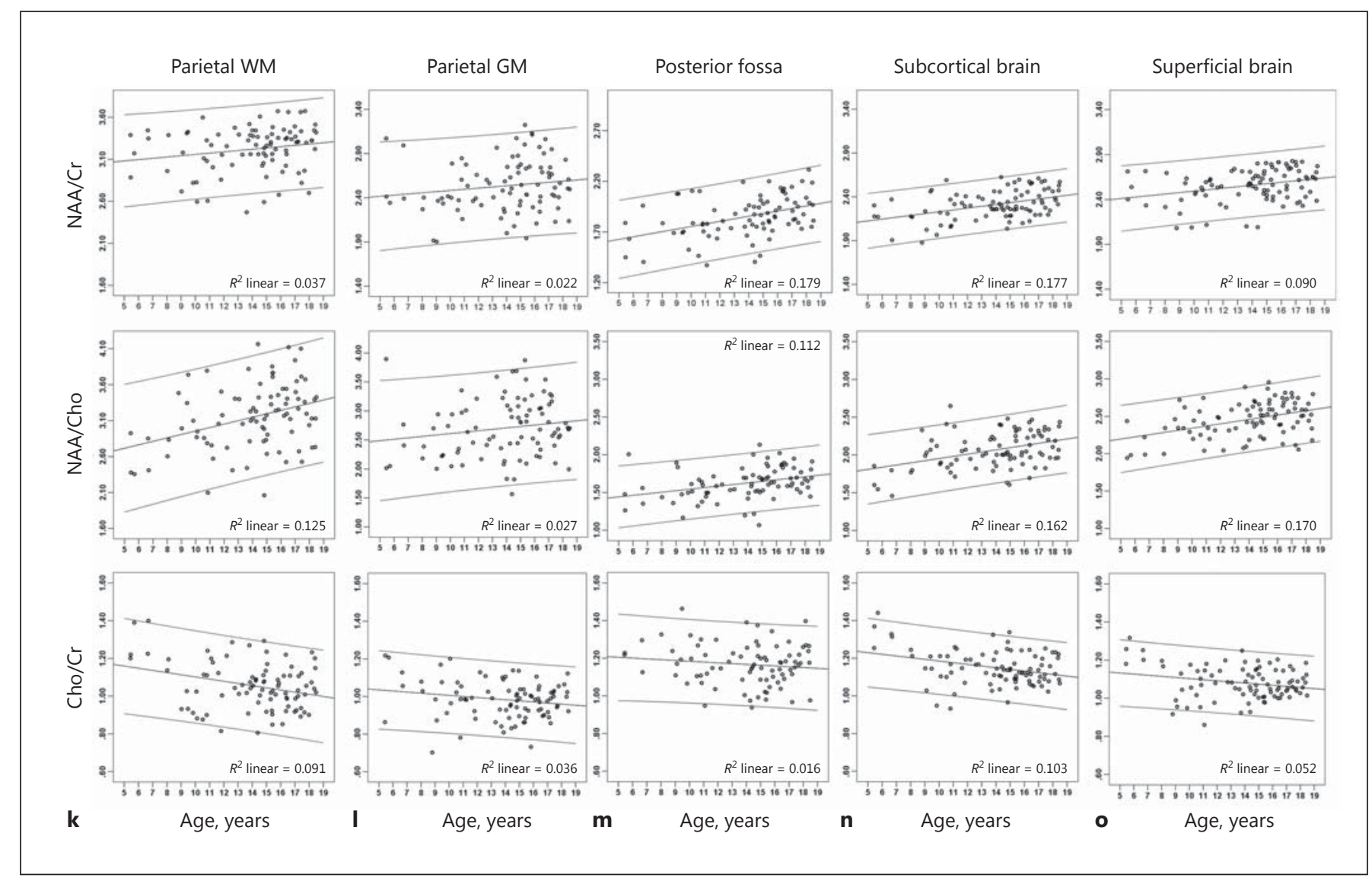

Fig. 6. Measured magnetic resonance spectroscopic imaging (MRSI) parameters for N-acetylaspartate (NAA)/ creatine (Cr), NAA/choline (Cho) and Cho/Cr for 12 brain regions and 3 combined regions; subcortical (basal ganglia + thalami + corpus callosum), superficial brain (temporal + parietal + frontal white matter [WM] and gray matter $[\mathrm{GM}]$ ), and posterior fossa (brain stem + cerebellum) versus age in years. The linear regression fits, $R^{2}$ value, and $95 \%$ confidence intervals are shown.

fidence intervals, and $R^{2}$ values (Fig. 5a-i). We found that significant increases in mean FA and decreases in mean diffusivity with age were regionally dependent. Results of multiple regression analyses to evaluate significant changes with age for DTI and MRSI parameters are shown in Table 2.

Several regions showed significant maturational changes for several DTI parameters; however, for this age range, the percent change tended to be small. In the posterior fossa which included only the BS/pons region (BS; Fig. 5a), the largest change showed a $9 \%$ decrease in $\mathrm{RD}$ $(p=0.014)$. In the subcortical region (Fig. 5h) that included the combined BG (Fig. 5b), TH (Fig. 5c), and CC (Fig. 5d), the largest combined percent change was an increased FA of $10 \%$ primarily due to increases in the BG $(12.7 \%)$ and $\mathrm{TH}(9 \%)$. Although no change in FA was

DTI and MRSI Brain Analysis in Normal Children seen in the CC, a $15 \%$ decrease in $\mathrm{RD}$ was recorded. Maturational changes in the superior brain regions (Fig. 5i) (i.e., temporal WM, Fig. 5e; frontal WM, Fig. 5f; and parietal WM, Fig. $5 \mathrm{~g}$ ) were observed in which the largest combined percent change was a decreased ADC (-9.7\%) due to a decrease in all $3 \mathrm{WM}$ regions. A $12.6 \%$ decrease in $\mathrm{RD}$ was also seen in the temporal WM.

\section{Normal Developmental Patterns of Metabolite Ratios}

Metabolite changes with age are shown in graphs for individual and combined regions with linear fits, confidence intervals, and $R^{2}$ values (Fig. 6a-o). Several regional metabolite ratios showed significant changes with age using multiple regression analyses (Table 2). Overall, metabolite ratios showed greater percent maturational changes than the DTI parameters. The largest percent

Dev Neurosci 2017;39:413-429 
Table 3. Mean metabolite ratios $\pm 1 \mathrm{SD}$ for age groups

\begin{tabular}{|c|c|c|c|c|c|c|}
\hline & \multicolumn{2}{|l|}{$\mathrm{NAA} / \mathrm{Cr}$} & \multicolumn{2}{|l|}{ NAA/Cho } & \multicolumn{2}{|l|}{ Cho/Cr } \\
\hline & $<12$ years & $\geq 12$ years & $<12$ years & $\geq 12$ years & $<12$ years & $\geq 12$ years \\
\hline BG & $2.01 \pm 0.20$ & $2.12 \pm 0.21^{*}$ & $1.91 \pm 0.31$ & $1.99 \pm 0.26$ & $1.1 \pm 0.13$ & $1.07 \pm 0.08$ \\
\hline BS & $1.80 \pm 0.20$ & $1.99 \pm 0.20^{* * *}$ & $1.43 \pm 0.20$ & $1.61 \pm 0.19^{* * *}$ & $1.28 \pm 0.14$ & $1.25 \pm 0.13$ \\
\hline $\mathrm{CB}$ & $1.60 \pm 0.34$ & $1.66 \pm 0.32$ & $1.81 \pm 0.28$ & $1.92 \pm 0.33$ & $0.89 \pm 0.13$ & $0.86 \pm 0.10$ \\
\hline $\mathrm{CC}$ & $2.73 \pm 0.23$ & $2.90 \pm 0.21^{* *}$ & $2.28 \pm 0.42$ & $2.47 \pm 0.29 *$ & $1.27 \pm 0.16$ & $1.21 \pm 0.11$ \\
\hline FGM & $2.40 \pm 0.33$ & $2.43 \pm 0.28$ & $2.23 \pm 0.38$ & $2.23 \pm 0.35$ & $1.14 \pm 0.12$ & $1.13 \pm 0.10$ \\
\hline FWM & $2.63 \pm 0.34$ & $2.73 \pm 0.31$ & $2.40 \pm 0.40$ & $2.50 \pm 0.35$ & $1.17 \pm 0.16$ & $1.13 \pm 0.10$ \\
\hline OGM & $2.40 \pm 0.40$ & $2.50 \pm 0.35^{*}$ & $2.60 \pm 0.56$ & $2.87 \pm 0.49^{* *}$ & $0.98 \pm 0.11$ & $0.89 \pm 0.13$ \\
\hline PGM & $2.45 \pm 0.28$ & $2.56 \pm 0.30$ & $2.58 \pm 0.48$ & $2.75 \pm 0.51$ & $1.00 \pm 0.13$ & $0.97 \pm 0.09$ \\
\hline PWM & $3.12 \pm 0.29$ & $3.26 \pm 0.25^{*}$ & $2.93 \pm 0.44$ & $3.23 \pm 0.44^{* *}$ & $1.09 \pm 0.16$ & $1.04 \pm 0.11$ \\
\hline TGM & $1.99 \pm 0.21$ & $1.98 \pm 0.22$ & $1.93 \pm 0.26$ & $1.94 \pm 0.22$ & $1.05 \pm 0.10$ & $1.05 \pm 0.09$ \\
\hline TWM & $2.47 \pm 0.22$ & $2.47 \pm 0.20$ & $2.09 \pm 0.19$ & $2.19 \pm 0.24$ & $1.18 \pm 0.12$ & $1.15 \pm 0.09$ \\
\hline $\mathrm{TH}$ & $2.12 \pm 0.20$ & $2.18 \pm 0.20$ & $1.80 \pm 0.24$ & $1.89 \pm 0.24$ & $1.21 \pm 0.15$ & $1.18 \pm 0.09$ \\
\hline SC & $2.22 \pm 0.18$ & $2.35 \pm 0.15^{* *}$ & $1.89 \pm 0.28$ & $2.10 \pm 0.21^{*}$ & $1.18 \pm 0.12$ & $1.14 \pm 0.08$ \\
\hline $\mathrm{PF}$ & $1.74 \pm 0.21$ & $1.90 \pm 0.18^{* *}$ & $1.53 \pm 0.20$ & $1.67 \pm 0.20^{* *}$ & $1.19 \pm 0.11$ & $1.16 \pm 0.11$ \\
\hline SB & $2.47 \pm 0.18$ & $2.59 \pm 0.17^{* *}$ & $2.33 \pm 0.22$ & $2.51 \pm 0.22^{* *}$ & $1.09 \pm 0.12$ & $1.07 \pm 0.07$ \\
\hline Total & $2.33 \pm 0.16$ & $2.47 \pm 0.13^{* * *}$ & $2.15 \pm 0.21$ & $2.33 \pm 0.19^{* * *}$ & $1.13 \pm 0.11$ & $1.10 \pm 0.07$ \\
\hline
\end{tabular}

See Tables 1 and 2 for abbreviations. ${ }^{*} p<0.05,{ }^{* *} p<0.01,{ }^{* * *} p<0.001$.

changes were seen with NAA/Cho ratios which may be expected since NAA and Cho are changing in opposite directions. The largest significant percent increase in NAA/Cr was seen in the BS (18.8\%; Fig. $6 \mathrm{~b})$ followed by the subcortical regions in the BG (11.9\%; Fig. 6e), CC (8.9\%; Fig. $6 \mathrm{~g})$, and the TH (6.0\%; Fig. $6 \mathrm{f})$. None of the superficial brain, WM, or GM regions showed a significant increase in NAA/Cr with age. The largest percent decreases in $\mathrm{Cho} / \mathrm{Cr}$ were seen in the posterior superior brain regions of the occipital GM (-14.6\%; Fig. 6j) and the parietal WM (-12.5\%; Fig. 6k).

When subjects were stratified into two groups based on an age cutoff of 12 years determined empirically with graphical methods, we found that the mean values of all ratios were within $1 \mathrm{SD}$ between the two groups even when statistical differences were highly significant (Table 3). Overall, the mean NAA/Cr ratios were slightly higher and the mean $\mathrm{Cho/Cr}$ ratios were slightly lower for the older age group as would be expected with brain maturation.

\section{Correlations between DTI Parameters and Metabolite Ratios}

We found consistent, significant $(p<0.01)$, but weakly positive correlations $(r=0.228-0.329)$ between NAA ratios and mean FA in the BS, BG, and CC regions. No significant correlations between thalamic DTI and metabolite ratios were found. In WM regions, no correlations between mean FA and NAA ratios were found, but significant $(p<0.01)$, weakly negative correlations $(r=$ -0.272 to -0.357 ) were found between NAA ratios and $\mathrm{WM} A \mathrm{ADC}$ and $\mathrm{AD}$ measurements.

\section{Discussion}

Although we are reporting only normative data in this study, these methods were developed to ultimately compare data between healthy controls and TBI patients. We decided to take this approach since a key limitation of the TBSS method is the requirement of registering DTI tracts between subjects - for example, either healthy controls over time (for neurodevelopment) or controls to TBI patients (for neurodegeneration) - while handling tract deformations robustly. Although TBSS skeletons are claimed to be alignment invariant [25], skeletal voxelwise comparison will suffer from the possibility of oneto-many mapping between two skeletons, accounting for fiber crossings, as well as branching, specifically between the control template and severely injured (deformed) brains $[34,35]$. Also, as pointed out in neurodevelopmental studies, tract-based registration and comparison might 
suffer from cortical boundaries due to inherent WM variations, specifically in children $[8,26]$. For large deformations in severe TBI $[35,36]$, such tract-wise registration might not work well, leading to considerable errors, and hence manual placement of bilateral regions of interest on relevant tracts is often adopted as the last resort [35, 37]. The current method might be more robust in detecting inherent subtle variation in developing brains as its alignment is based on much larger regions (brain anatomy compared to voxels or skeletons or WM tracts) including both WM and GM tissue.

To detect the WM regions, the current study utilized a mid-level FA threshold $>0.2$ that has recently been reported to be inclusive enough for robust statistical comparison $[2,28,38-40]$ and has also been validated in our study (Fig. 3). When FA constraints are too relaxed (e.g., FA $>0.15)[26,41]$, small subtracts may be included leading to false-positive results. On the other hand, restrictive FA constraints, e.g., FA $>0.25[28,31,42]$ or FA $>0.3$ [25], exclude WM regions and hence suffer from high falsenegative rates. Although some research studies support a more inclusive FA threshold in analysis of neonatal WM [43], demarcation between adults and neonates is uncertain as WM maturation is found to decelerate after 2 years of age [8].

For anatomic parcellation of DTI data, previous studies have adopted a variety of methods - including (1) manual demarcation of selected DTI anatomy [38, 40], (2) utilization of a T1 space atlas directly in DTI space [24], (3) estimation of indigenous DTI templates of the concerned age group from TBSS [26] or tractographybased data [39], and (4) utilization of established adult DTI atlases even for pediatric data with an assumption of less structural difference after 2 years of age $[1,8,31]$. The current work adopts yet another option by registering the pediatric subject's T1/T2 data with an established pediatric T1/T2 atlas and then registering the subject's T1/T2 with its own DTI b0 data to map T1/T2 anatomy/tissue information into the subject's DTI coordinate space. This is probably a more logical approach because in the first stage of registration, ages are comparable and the MR techniques are the same, only the subjects are different. While in the second stage of registration, the subject and thus the age are the same, just the MR technique is different $[2,26]$.

MRS data complements DTI and other MRI data in multimodal studies $[41,44,45]$. Anatomic parcellation of MRS data has been performed by prior knowledge-based manual bilateral region of interest placement [35], ad hoc heuristics [41], or an established (Harvard-Oxford) atlas

DTI and MRSI Brain Analysis in Normal Children
[46]. One recent work reports explicit co-alignment of MRSI data, first to corresponding high-resolution T1 data and then to a T1 atlas space, using additional lowresolution $\mathrm{T} 1$ overlay data with the same geometry as the MRSI data explicitly collected for this purpose [45]. Another study on 4 volunteers used a methodology based on linear displacement, angular rotation, and pixel-based interpolation of DTI images to co-register MRSI voxels to DTI images [47]. In this study, a similar approach was used with the image geometry of different acquisitions (MRSI, T1, T2, and DTI) being directly compared to determine image transformation parameters, map anatomical information from T1/T2 atlases to the MRSI coordinates, and fuse anatomy-specific MRS data with DTI. This was done to give us the capability to directly compare metabolite with DTI maturational changes in the same anatomic voxels or regions.

In agreement with other studies, we found evidence for WM maturation throughout various brain regions from childhood through adolescence using DTI $[3,6,48-50]$. For children and adolescents included in this study (age range 5-18 years), most age-related changes in DTI parameters were $<10 \%$, also in agreement with other authors showing subtle changes after age 5 years. Our methods did not include measurements of specific WM tracts since we were trying to directly compare regions between MRSIsized voxels and DTI parameters within those voxels. We found that the largest significant increases in FA with age were in the subcortical regions of BG and TH (average of $10.9 \%$; Table 2), which may reflect increasing myelination, axonal density, and diameter as well as overall maturation of the BG-TH-CC pathways [48]. We also observed small but significant decreases in $\mathrm{AD}$, a measure of axonal volume, in the superior brain WM regions (frontal, parietal, and temporal WM) and the CC. More significant decreases in $\mathrm{RD}$, a measure of myelination, were seen in the BG, CC, temporal WM, and frontal WM with associated increases in FA suggesting that decreases in $\mathrm{RD}$ contributed more to increasing FA in brain maturation. Decreases in $\mathrm{AD}$ and $\mathrm{RD}$ may reflect increasing compactness and myelination, respectively [51]. In agreement with other studies $[8,52,53]$, we found that ADC, an indicator of the total amount of diffusion, decreased with age in all regions except the TH. Although we show that changes in DTI indices are relatively small in the 5- to 18-year age range, it may be necessary to take these small regional changes into consideration in order to detect subtle abnormalities especially in diseases where the brain may be mildly affected or when longitudinal studies (e.g., clinical trials) are performed over extended time periods.

Dev Neurosci 2017;39:413-429

DOI: $10.1159 / 000475545$ 
Changes in metabolite ratios were found in normal subjects in the age range studied and are thought to be related to normal maturational changes. In general, the metabolite changes are a result of both neuronal differentiation seen as increasing NAA levels and ongoing myelination seen as decreasing Cho levels [18]. The NAA/Cr ratios showed the highest percent change in the BS followed by the subcortical regions and no change with age in the superior GM and WM regions. However, this study is reporting ratios rather than individual metabolite levels, and we must take into consideration that the reason we are not seeing changes in the superior brain regions is not because maturation is not taking place but because $\mathrm{Cr}$ is also increasing. Particularly since the NAA/Cho ratios showed a greater significant increase in all regions (except the parietal and temporal GM, and cerebellum) indicating that these changes appear to be related to both increasing NAA (as NAA/Cr and NAA/Cho) and declining Cho (as Cho/Cr). Other quantitative metabolite studies report a consistent subtle total $\mathrm{Cr}$ increase in the posteromedial cortex [54] and parietal/occipital GM regions [18] through adolescence. In our study, Cho/Cr ratio changes were greatest in the parietal WM and occipital GM regions, which would support that changes were occurring due to decreasing Cho as well as increasing Cr. All other superior brain regions showed decreasing $\mathrm{Cho} / \mathrm{Cr}$ ratios, which may be associated with increasing myelination. It is interesting to note that frontal regions showed less change than posterior regions, which follows prior brain maturation studies that emphasize a complex posteriorto-anterior gradient through adolescence and into adulthood [55].

Although we show significant spectroscopic changes with age, the rates of change are subtle compared to changes seen from birth through early childhood and agree with several other reports of normal metabolite changes in children and adolescents $[19,20]$. In addition, we found that metabolite ratios varied slightly more from 5 to 12 years of age than from 12 to 18 years of age (Table 3), which seems to be a natural division of age used as neurobehavioral landmarks in clinical neuropsychological studies [18]. However, when comparing mean values between the two age groups, no regions showed significant differences in Cho/Cr ratios, and only a few regions showed significant differences with NAA ratios. In addition, means between groups were within 1 SD demonstrating that metabolite ratio changes are subtle in the age range studied. Nevertheless, these means demonstrate slightly higher NAA ratios and lower $\mathrm{Cho/Cr}$ ratios for the older age group, which is expected with brain mat- uration and can be used for comparison with other pediatric cohorts.

In this study, we did not observe any strong correlations between regional DTI parameters and corresponding regional metabolite ratios. This may be a result of comparing averaged DTI measures from WM contained within large brain regions rather than specific WM tracks. One study in healthy adult volunteers using VBA and color-coded correlation maps showed a strong positive correlation between quantitative NAA measurements and FA [45] only in the superior longitudinal fasciculus while another tractography study showed strong correlation with the uncinate fasciculus [41]. These findings may be attributed to greater neuronal density and a greater structural organization in specific WM tracts. Another study using similar methods to our own showed a strong negative correlation of ADC with NAA in an area containing the superior longitudinal fasciculus in 4 adult volunteers; however, correlations between other DTI measures and metabolites showed nonsignificant trends only [47]. We also saw significant weak-to-moderate inverse correlations with $\mathrm{ADC}$ in WM regions. This may represent diffusion restriction of water in WM as a result of increased neuronal integrity. The thalamus was the only region in our study that showed nonsignificant no correlation between MRSI and DTI. A previous study [45] attributed lack of correlation in the thalamus to the presence of crossing fibers causing the net $\mathrm{FA}$ to equal nearly 0 in the thalamus.

\section{Limitations}

Both DTI and MRSI acquisitions are prone to various artifacts particularly in the pediatric population, including patient motion and signal-to-noise sensitivity. Since subjects in this study were at least 5 years old, we were able to scan all subjects without sedation. We also took the time to familiarize the children within the scanner environment to make them more comfortable before scanning. Images and spectra were checked for motion and repeated if necessary before the patient was released, and studies were rejected if the subject was not able to hold still. Also, the signal-to-noise ratio was similar in all patients since subjects were old enough that we did not need to change acquisition voxel size due to small head size. Partial volume effects can be pronounced in MRSI acquisitions. In this study, we used segmentation software to assign voxel tissue percentages in order to limit the partial volume effects. Rejection of outliers and 
cleaning of datasets with missing entries $[17,46,56]$ were used in the current study. DTI images can also suffer from pulsation artifacts, hardware instability, and susceptibility artifacts at tissue-air interfaces and particularly due to metal; therefore, children with braces were not included in the study.

Volumetric registration across multiple MRI and MRSI acquisition spaces of the subject and atlas data with different geometry and resolution is challenging. One limitation of the current method is suboptimal brain parsing in the subcortical region that is inherent in T1-based brain atlases. This can be improved by utilizing a T1-atlas for cortical regions and a T2-atlas for subcortical regions for brain parsing and subsequently merging them, at the cost of an additional registration step between subjects' T1WI and T2WI data. In the future when using this method for patients with significant brain injury (as in severe TBI), a modification of the current registration method may be needed to handle image distortion caused by deformities or large hemorrhages or contusions such as temporary removal of the distorted brain region before volumetric registration. The computed image transformation matrix can then be utilized to reinsert the injured region and then estimate its anatomy-specific statistics. In addition, methods to display subject-specific abnormalities will be utilized for whole brain DTI statistics based on $z$-score distributions [57]. Such complexities would be handled in our future work.

\section{Conclusions}

We report on an automated computational method to anatomically fuse and directly compare data from whole brain DTI and 3D MRSI acquisitions from pediatric control subjects. We also provide extensive age- and regionspecific normative MR diffusion and spectroscopic metabolite ranges that show brain maturation profiles and are requisite for detecting abnormalities in an injured or diseased population. Our particular interest is related to pediatric TBI, but the normative values presented in this paper can be applied for comparison to any injured or diseased pediatric population when similar acquisition techniques are used. Another unique aspect of this study is fusing spectroscopic and DTI data for direct regional comparisons.

\section{Acknowledgments}

The authors would like to acknowledge and thank our MR technologists and pediatric nurse managers whose diligent work made this project possible. This work was supported by the National Institute of Health (NINDS 5R01NS05400). The content is solely the responsibility of the authors and does not necessarily represent the official views of the NINDS or the NIH.

\section{Disclosure Statement}

The authors have no conflict of interest.

\section{References}

1 Loh KB, Ramli N, Tan LK, Roziah M, Rahmat $\mathrm{K}$, Ariffin H: Quantification of diffusion tensor imaging in normal white matter maturation of early childhood using an automated processing pipeline. Eur Radiol 2012;22: 1413-1426.

2 Qiu D, Tan LH, Zhou K, Khong PL: Diffusion tensor imaging of normal white matter maturation from late childhood to young adulthood: voxel-wise evaluation of mean diffusivity, fractional anisotropy, radial and axial diffusivities, and correlation with reading development. Neuroimage 2008;41:223-232.

3 Yoshida S, Oishi K, Faria AV, Mori S: Diffusion tensor imaging of normal brain development. Pediatr Radiol 2013;43:15-27.

4 Mukherjee P, Miller JH, Shimony JS, Philip JV, Nehra D, Snyder AZ, et al: Diffusion-tensor MR imaging of gray and white matter development during normal human brain maturation. AJNR Am J Neuroradiol 2002;23: 1445-1456.
5 Huang H, Zhang J, Wakana S, Zhang W, Ren $\mathrm{T}$, Richards LJ, et al: White and gray matter development in human fetal, newborn and pediatric brains. Neuroimage 2006;33:27-38.

6 Krogsrud SK, Fjell AM, Tamnes CK, Grydeland H, Mork L, Due-Tønnessen P, et al: Changes in white matter microstructure in the developing brain - a longitudinal diffusion tensor imaging study of children from 4 to 11 years of age. Neuroimage 2016;124:473486.

7 Uda S, Matsui M, Tanaka C, Uematsu A, Miura K, Kawana I, Noguchi K: Normal development of human brain white matter from infancy to early adulthood: a diffusion tensor imaging study. Dev Neurosci 2015;37:182-194.

8 Faria AV, Zhang J, Oishi K, Li X, Jiang H, Akhter K, et al: Atlas-based analysis of neurodevelopment from infancy to adulthood using diffusion tensor imaging and applications for automated abnormality detection. Neuroimage 2010;52:415-428.
9 Bluml S, Moreno A, Hwang JH, Ross BD: 1-(13)C glucose magnetic resonance spectroscopy of pediatric and adult brain disorders. NMR Biomed 2001;14:19-32.

10 Vorona GA, Berman JI: Review of diffusion tensor imaging and its application in children. Pediatr Radiol 2015;45(suppl 3):S375-S381.

11 Rollins NK: Clinical applications of diffusion tensor imaging and tractography in children. Pediatr Radiol 2007;37:769-780.

12 Wahl M, Strominger ZA, Wakahiro M, Jeremy RJ, Mukherjee P, Sherr EH: Diffusion tensor imaging of Aicardi syndrome. Pediatr Neurol 2010;43:87-91.

13 Ulmer S, Backens M, Ahlhelm FJ: Basic principles and clinical applications of magnetic resonance spectroscopy in neuroradiology. J Comput Assist Tomogr 2016;40:1-13.

14 Ashwal S, Tong KA, Ghosh N, Bartnik-Olson B, Holshouser BA: Application of advanced neuroimaging modalities in pediatric traumatic brain injury. J Child Neurol 2014;29: 1704-1717. 
15 Ross B, Bluml S: Magnetic resonance spectroscopy of the human brain. Anat Rec 2001; 265:54-84.

16 Huppi PS, Maier SE, Peled S, Zientara GP, Barnes PD, Jolesz FA, Volpe JJ: Microstructural development of human newborn cerebral white matter assessed in vivo by diffusion tensor magnetic resonance imaging. Pediatr Res 1998;44:584-590.

17 Kreis R, Hofmann L, Kuhlmann B, Boesch C, Bossi E, Hüppi PS: Brain metabolite composition during early human brain development as measured by quantitative in vivo ${ }^{1} \mathrm{H}$ magnetic resonance spectroscopy. Magn Reson Med 2002;48:949-958.

18 Bluml S, Wisnowski JL, Nelson MD Jr, Paquette L, Gilles FH, Kinney HC, Panigrahy A: Metabolic maturation of the human brain from birth through adolescence: insights from in vivo magnetic resonance spectroscopy. Cereb Cortex 2013;23:2944-2955.

19 Pouwels PJ, Brockmann K, Kruse B, Wilken B, Wick M, Hanefeld F, Frahm J: Regional age dependence of human brain metabolites from infancy to adulthood as detected by quantitative localized proton MRS. Pediatr Res 1999; 46:474-485.

20 Holshouser BA, Ashwal S, Luh GY, Shu S, Kahlon S, Auld KL, Tomasi LG, Perkin RM, Hinshaw DB Jr: Proton MR spectroscopy after acute central nervous system injury: outcome prediction in neonates, infants, and children. Radiology 1997;202:487-496.

21 Dinov ID, Petrosyan P, Liu Z, Eggert P, Zamanyan A, Torri F, Macciardi F, et al: The perfect neuroimaging-genetics-computation storm: collision of petabytes of data, millions of hardware devices and thousands of software tools. Brain Imaging Behav 2014;8:311322.

22 Goh SY, Irimia A, Torgerson CM, Horn JD: Neuroinformatics challenges to the structural, connectomic, functional and electrophysiological multimodal imaging of human traumatic brain injury. Front Neuroinform 2014; 8:19.

23 Akil H, Martone ME, Van Essen DC: Challenges and opportunities in mining neuroscience data. Science 2011;331:708-712.

24 Munoz Maniega S, Lymer GK, Bastin ME, Marjoram D, Job DE, Moorhead TW, et al: A diffusion tensor MRI study of white matter integrity in subjects at high genetic risk of schizophrenia. Schizophr Res 2008;106:132139.

25 Smith SM, Jenkinson M, Johansen-Berg H, Rueckert D, Nichols TE, Mackay CE, et al: Tract-based spatial statistics: voxelwise analysis of multi-subject diffusion data. Neuroimage 2006;31:1487-1505.

26 Singh M, Jeong J, Hwang D, Sungkarat W, Gruen P: Novel diffusion tensor imaging methodology to detect and quantify injured regions and affected brain pathways in traumatic brain injury. Magn Reson Imaging 2010;28:22-40
27 Amarreh I, Meyerand ME, Stafstrom C, Hermann BP, Birn RM: Individual classification of children with epilepsy using support vector machine with multiple indices of diffusion tensor imaging. Neuroimage Clin 2014;4: 757-764.

28 Li M, Ratnanather JT, Miller MI, Mori S Knowledge-based automated reconstruction of human brain white matter tracts using a path-finding approach with dynamic programming. Neuroimage 2014;88:271-281.

29 Niogi SN, Mukherjee P, McCandliss BD: Diffusion tensor imaging segmentation of white matter structures using a reproducible objective quantification scheme (ROQS). Neuroimage 2007;35:166-174.

30 Van Hecke W, Sijbers J, De Backer S, Poot D, Parizel PM, Leemans A: On the construction of a ground truth framework for evaluating voxel-based diffusion tensor MRI analysis methods. Neuroimage 2009;46:692-707.

31 Cubon VA, Putukian M, Boyer C, Dettwiler A: A diffusion tensor imaging study on the white matter skeleton in individuals with sports-related concussion. J Neurotrauma 2011;28:189-201.

32 Pierpaoli C, Basser PJ: Toward a quantitative assessment of diffusion anisotropy. Magn Reson Med 1996;36:893-906.

33 Basser PJ, Jones DK: Diffusion-tensor MRI: theory, experimental design and data analysis - a technical review. NMR Biomed 2002; 15:456-467.

34 Jones DK, Knosche TR, Turner R: White matter integrity, fiber count, and other fallacies the do's and don'ts of diffusion MRI. Neuroimage 2013;73:239-254.

35 Tollard E, Galanaud D, Perlbarg V, SanchezPena P, Le Fur Y, Abdennour L, et al: Experience of diffusion tensor imaging and ${ }^{1} \mathrm{H}$ spectroscopy for outcome prediction in severe traumatic brain injury: preliminary results. Crit Care Med 2009;37:1448-1455.

36 Sidaros A, Skimminge A, Liptrot MG, Sidaros $\mathrm{K}$, Engberg AW, Herning M, et al: Long-term global and regional brain volume changes following severe traumatic brain injury: a longitudinal study with clinical correlates. Neuroimage 2009;44:1-8.

37 Kumar R, Husain M, Gupta RK, Hasan KM, Haris M, Agarwal AK, et al: Serial changes in the white matter diffusion tensor imaging metrics in moderate traumatic brain injury and correlation with neuro-cognitive function. J Neurotrauma 2009;26:481-495.

38 Wu TC, Wilde EA, Bigler ED, Li X, Merkley TL, Yallampalli R, et al: Longitudinal changes in the corpus callosum following pediatric traumatic brain injury. Dev Neurosci 2010; 32:361-373.
39 Verhoeven JS, Sage CA, Leemans A, Van Hecke W, Callaert D, Peeters R, et al: Construction of a stereotaxic DTI atlas with full diffusion tensor information for studying white matter maturation from childhood to adolescence using tractography-based segmentations. Hum Brain Mapp 2010;31:470486.

40 Treble A, Hasan KM, Iftikhar A, Stuebing KK, Kramer LA, Cox CS Jr: Working memory and corpus callosum microstructural integrity after pediatric traumatic brain injury: a diffusion tensor tractography study. J Neurotrauma 2013;30:1609-1619.

41 Sato T, Maruyama N, Hoshida T, Minato K: Correlation between diffusion tensor tractography and proton MR spectroscopy in normal controls. Conf Proc IEEE Eng Med Biol Soc 2013;2013:515-518.

42 Calabrese E, Du F, Garman RH, Johnson GA, Riccio C, Tong LC, Long JB: Diffusion tensor imaging reveals white matter injury in a rat model of repetitive blast-induced traumatic brain injury. J Neurotrauma 2014;31:938950.

43 Vassar RL, Barnea-Goraly N, Rose J: Identification of neonatal white matter on DTI: influence of more inclusive thresholds for atlas segmentation. PLoS One 2014;9:e115426.

44 Talavage TM, Nauman EA, Leverenz LJ: The role of medical imaging in the recharacterization of mild traumatic brain injury using youth sports as a laboratory. Front Neurol 2015;6:273

45 Hao X, Xu D, Bansal R, Dong Z, Liu J, Wang $\mathrm{Z}$, et al: Multimodal magnetic resonance imaging: the coordinated use of multiple, mutually informative probes to understand brain structure and function. Hum Brain Mapp 2013;34:253-271.

46 Narayana PA, Yu X, Hasan KM, Wilde EA Levin HS, Hunter JV, et al: Multi-modal MRI of mild traumatic brain injury. Neuroimage Clin 2015;7:87-97.

47 Irwan R, Sijens PE, Potze JH, Oudkerk M: Correlation of proton MR spectroscopy and diffusion tensor imaging. Magn Reson Imaging 2005;23:851-858.

48 Barnea-Goraly N, Menon V, Eckert M, Tamm L, Bammer R, Karchemskiy A, et al: White matter development during childhood and adolescence: a cross-sectional diffusion tensor imaging study. Cereb Cortex 2005; 15 : $1848-1854$.

49 Mukherjee P, Miller JH, Shimony JS, Conturo TE, Lee BC, Almli CR, McKinstry RC: Normal brain maturation during childhood: developmental trends characterized with diffusion-tensor MR imaging. Radiology 2001; 221:349-358.

50 Asato MR, Terwilliger R, Woo J, Luna B: White matter development in adolescence: a DTI study. Cereb Cortex 2010;20:2122-2131. 
51 Pierpaoli C, Barnett A, Pajevic S, Chen R, Penix LR, Virta A, Basser P: Water diffusion changes in Wallerian degeneration and their dependence on white matter architecture. Neuroimage 2001;13:1174-1185.

52 Bonekamp D, Nagae LM, Degaonkar M, Matson M, Abdalla WM, Barker PB, et al: Diffusion tensor imaging in children and adolescents: reproducibility, hemispheric, and agerelated differences. Neuroimage 2007;34 733-742.
53 Snook L, Paulson LA, Roy D, Phillips L, Beaulieu C: Diffusion tensor imaging of neurodevelopment in children and young adults. Neuroimage 2005;26:1164-1173.

54 Degnan AJ, Ceschin R, Lee V, Schmithorst VJ, Blüml S, Panigrahy A: Early metabolic development of posteromedial cortex and thalamus in humans analyzed via in vivo quantitative magnetic resonance spectroscopy. J Comp Neurol 2014;522:3717-3732.

55 Toga AW, Thompson PM, Sowell ER: Mapping brain maturation. Trends Neurosci 2006;29:148-159.
56 Maudsley AA, Govind V, Levin B, Saigal G, Harris L, Sheriff S: Distributions of magnetic resonance diffusion and spectroscopy measures with traumatic brain injury. J Neurotrauma 2015;32:1056-1063.

57 Mayer AR, Bedrick EJ, Ling JM, Toulouse T, Dodd A: Methods for identifying subjectspecific abnormalities in neuroimaging data. Hum Brain Mapp 2014;35:5457-5470. 